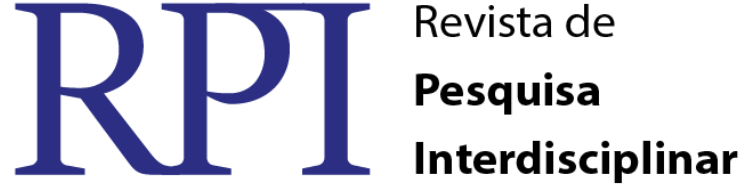

\section{REPRESENTAÇÕES SOCIAIS SOBRE O TABAGISTA NA SAÚDE}

\author{
Sônia Cardoso Moreira Garcia ${ }^{1}$ \\ Cristina Novikoff ${ }^{2}$
}

\begin{abstract}
RESUMO
$\mathrm{O}$ artigo versa sobre as representações sociais de tabagistas para profissionais que atuam no Programa Governamental para Controle do Tabagismo - PGCT de Resende. O tabagismo, segundo a Organização Mundial de Saúde (OMS) é considerado uma doença crônica transmissível através da propaganda e publicidade. É fator de risco para cerca de 50 doenças, entre elas as associadas às cardiovasculares e respiratórias. No Brasil, os programas que tratam do problema são: Programa de Saúde da Família - PSF, Estratégia de Saúde da Família - ESF e o Saúde da Família - SF, designadas, aqui, pela sigla PSF/ESF/SF. Ao considerarmos que somente $20 \%$ dos tabagistas aderem ao Programa, suspeitamos de que a forma como estes são representados pelos profissionais poderiam estar dificultando esta adesão. A Teoria das Representações Sociais (MOSCOVICI, 1979) nos possibilita compreender como os conhecimentos e valores sobre o outro inferem nas práticas cotidianas. Assim, objetivamos compreender as representações sociais de tabagistas para os profissionais do PSF/ESF/SF de Resende/RJ. A pesquisa foi norteada pelas dimensões Novikoff (2010) de natureza qualitativa do tipo descritiva de campo, com coleta de dados por meio de Técnica Projetiva junto aos citados profissionais. Os resultados sinalizam as representações sociais sobre o tabagista com ênfase nas características negativas (97\%) para os profissionais que trabalham em PSFs. Portanto, nosso estudo no enfrentamento do tabagismo oferece recursos, dentro de uma visão humanizada, que torna o tratamento em si, viável e atual.
\end{abstract}

Palavras-chave: Representações sociais; Saúde; Tabagismo.

\section{SOCIAL REPRESENTATIONS ON THE HEALTH TUBER}

\begin{abstract}
The article deals with the social representations of smokers for professionals who work in the Governmental Program for Tobacco Control - PGCT de Resende. Smoking, according to the World Health Organization (WHO) is considered a chronic disease transmitted through advertising and publicity. It is a risk factor for about 50 diseases, including those associated with cardiovascular and respiratory diseases. In Brazil, the programs that deal with the problem are: Family Health Program PSF, Family Health Strategy - FHT and Family Health - SF, designated here by the acronym PSF / ESF / SF. When we consider that only $20 \%$ of smokers join the Program, we suspect that the way they are represented by professionals could be making this compliance difficult. The Theory of Social Representations (MOSCOVICI, 1979) enables us to understand how knowledge and values about the other derive from everyday practices. Thus, we aimed to understand the social representations of smokers for PSF / ESF / SF professionals from Resende / RJ. The research was guided by the Novikoff (2010) dimensions of qualitative nature of the descriptive type of field, with data collection

\footnotetext{
${ }^{1}$ Psicóloga e professora do curso de medicina do Centro Universitário de Volta Redonda. Mestre em Ensino das Ciências da Saúde e do Meio Ambiente - UniFOA e Pesquisadora Colaboradora LAGERES/CNPq(2010-2012) - Laboratório do Grupo de Estudos e Pesquisas em Representações Sociais na/para formação de professores.

${ }^{2}$ Professora Pd.D. Educação da Universidade Federal de Campina Grande - UFCG e líder do Laboratório do Grupo de Estudos e Pesquisas em Representações Sociais na/para Formação de Professores - LAGERES/CNPq/UFCG.
} 
by means of Projective Technique with the mentioned professionals. The results indicate the social representations about the smoker, with emphasis on the negative characteristics (97\%) for professionals working in PSFs. Therefore, our study in the face of smoking offers resources, within a humanized vision, that makes the treatment itself, viable and current.

Keywords: Social representations; Cheers; Smoking.

\section{INTRODUÇÃO}

O presente artigo trata das representações sociais do tabagista para profissionais de $\mathrm{PSF} / \mathrm{ESF} / \mathrm{SF}$, considerando a emergência de novos estudos favorecedores de intervenções pragmáticas no enfrentamento da doença com adoção de estratégias de adesão para tratamento e cessação do vício do tabaco.

No mundo, segundo dados da Organização Mundial de Saúde (OMS), um terço da população adulta fuma, ou seja, há, na terra, 1,2 bilhão de fumantes, surgindo, assim uma epidemia de tabagismo. E como um dos fatores de risco mais importantes para as Doenças e Agravos Não-Transmissíveis (DANT), o tabagismo é a principal causa de óbitos e enfermidades no Brasil, com aproximadamente 20 milhões em $2007^{1}$ (Iglesias, et al., 2007) e 24,6 milhões em $2008^{2}$ (Brasil, 2011). O número de fumantes reduziu, mas ainda continua sendo um agravo à saúde dos brasileiros.

Ao tomarmos os dados do Instituto Nacional de Câncer (INCA) ${ }^{3}$, relativos ao ano 2008, em que, no Brasil, 200 mil mortes anuais são causadas pelo tabagismo; 16\% da população brasileira adulta é fumante; a concentração de fumantes é maior entre as pessoas com menos de oito anos de estudo do que entre pessoas com oito ou mais anos de estudo; o cigarro brasileiro é o $6^{\circ}$ mais barato do mundo; cerca de $8 \%$ dos gastos com internação e quimioterapia no Sistema Único de Saúde são atribuídos a doenças relacionadas ao consumo do tabaco e; que somente com estes dois procedimentos, o governo gasta $\mathrm{R} \$ 338,6$ milhões para tratar doenças relacionadas ao vicio, afirmamos a importância do tema para projetos que visam uma resposta pragmática para o enfrentamento da doença.

Ao fazer um corte histórico entre o surgimento do tabaco e seus primeiros relatos científicos, observamos que foi a partir da década de 60, após mais de três milênios que o homem começa a relacionar o cigarro ao adoecimento do fumante de modo científico e com mais rigor ético. Assim, os estudos científicos tem favorecido a luta contra este vício milenar.

RPI Revista de Pesquisa Interdisciplinar, Cajazeiras, v. 1, Ed. Especial, 02-19, set/dez. de 2016. 
Atualmente existem inúmeros trabalhos comprovando os malefícios do tabagismo à saúde do fumante e do não fumante, mas que se encontra exposto à fumaça do cigarro ${ }^{4}$ (BRASIL, 2011).

Ao passar dos anos e em um processo de constatação dos malefícios do cigarro, lenta e progressivamente, patologias respiratórias, vasculares, oncológicas e muitas outras foram sendo relacionadas ao tabagismo que, de elegante, vai se tornando inimigo público em vários países ${ }^{5}$ Malbergier (2008).

No Brasil, foi a partir da década de 70 que se tornaram mais evidentes as manifestações organizadas para o controle do tabagismo ${ }^{6}$ (Brasil, 2001).

O programa de controle do tabagismo é uma estratégia preconizada pelo Ministério da Saúde e que propicia a recuperação do estado de saúde dos fumantes com comprometimentos advindos do vício, bem como a prevenção do início do uso do tabaco e de seus derivados nas diversas faixas etárias.

Em março de 2010, a partir do protocolo preconizado pelo Ministério da Saúde, foi implantado em Resende o Programa Municipal de Controle do Tabagismo (PMCT). Desde então foram atendidas, aproximadamente, 4.148 pessoas usuárias do tabaco e de seus derivados e o índice médio de cessação deste Programa é de $72 \%$.

O citado programa funciona de forma descentralizada, onde as Unidades de Saúde da atenção primária são as principais portas de entrada para o acesso ao tratamento. Além delas, algumas Unidades Mistas e Policlínicas possuem equipes capacitadas e com grupos implantados. Assim, temos um total de, aproximadamente, 28 pontos de atendimento do referido programa.

A questão de partida é quais são representações socias de tabagistas para profissionais das PSF/ESF/SF? Daí pensar como essas interferem na adesão do usuário do tabaco ao tratamento oferecido?

O objetivo deste artigo é apresentar parte do estudo realizado acerca das representações sociais de tabagistas para profissionais do PSF/ESF/SF de diferentes especialidades em Resende no estado do Rio de Janeiro, com a finalidade de contribuir na discussão do tema.

Neste sentido, partiu-se do pressuposto de que as representações sociais que os profissionais da saúde manifestam no processo de tratamento junto ao tabagista inferem na RPI Revista de Pesquisa Interdisciplinar, Cajazeiras, v. 1, Ed. Especial, 02-19, set/dez. de 2016. 
adesão do tratamento e no seu resultado. Daí estudou-se o problema a partir da Teoria das Representações Sociais ${ }^{8}$ de Moscovici $(1979,2003)$ que, considerando que as representações sociais se manifestam tanto pela linguagem como por imagens ${ }^{9}$ (MOSCOVICI, JODELET, 2003; VALA, 2004), optamos para o estudo empírico, por estas duas formas de manifestação dessas representações.

Enfim, entendemos que as representações sociais ${ }^{10}$, como salienta Arruda (2008), podem ser refeitas e reconstruídas. Diante disso, percebe-se, portanto, que não só se está diante de forças poderosas e instigadoras de condutas anunciadas nos, modelos de representações que foram aprendidos e apreendidos, mas, também, se está lidando com a força representacional emanada do próprio profissional, que em muitos casos, mesmo através do não dito ${ }^{11}$ Jodelet (2005), ignora o poder de sua visão do outro e do seu discurso na reconstrução de suas representações sobre o vício e a dependência, dificultando o tratamento do tabagismo.

\section{Metodologia}

Para o desenvolvimento da pesquisa ${ }^{3}$ pautamo-nos nas cinco Dimensões de pesquisa propostas por Novikoff $(2006 ; 2010)$, ou seja, um caminho metodológico que sustenta a pesquisa enquanto fenômeno político e intencional dialógico. Esta trajetória nos permite entender o processo político e científico do nosso trabalho. Político na mesma compreensão que Habermas descreve.

[..] pesquisa é sempre também fenômeno político, por mais que seja dotada de sofisticação técnica e se mascare neutra. Não se reduz a fenômeno político, mas nunca o desfaz de todo. Por isso vale dizer: sabemos mais o que interessa. $\mathrm{O}$ que explica, em parte, por que conhecemos muito mais como não mudar, já que a produção de conhecimentos está nas mãos de privilegiados. O desconforto pode ser gigante, quando se descobre, por exemplo, que a pesquisa social sobre pobreza cresceu muito, mas nada tem a ver com sua debelação. É difícil, talvez impossível, estabelecer uma correlação positiva entre o conhecimento da pobreza e o seu enfrentamento prático, embora não fosse impróprio constatar o inverso (HABERMAS, 1982, p.14 apud NOVIKOFF, 2010, p.214).

Ao entender como pressuposto que a Teoria das Representações Sociais nos permite compreender o pensamento social, ou seja, as representações sociais ou senso comum de um

\footnotetext{
${ }^{3}$ A pesquisa foi autorizada pelo Comitê de Ética em Pesquisa com Seres Humanos. CAAE: 08263213.4.0000.5237.

RPI Revista de Pesquisa Interdisciplinar, Cajazeiras, v. 1, Ed. Especial, 02-19, set/dez. de 2016.
} 
determinado grupo social, acredita-se que a investigação destas possibilita a identificação da forma como os profissionais da PSF/ESF/SF compreendem o tabagista.

Neste sentido, as representações sociais, enquanto fenômeno, ou seja, é tudo aquilo, seja sensação, sentimento ou ideia, que está presente na mente, seja registro na realidade ou da imaginação, merecem ser estudadas Santaella(1983). Representações sociais podem ser observadas por diferentes linguagens. Optamos pelo texto e o desenho, ambos possibilitam analisar dois processos: primeiro, compreender a subjetividade; segundo, pensar formas de intervenção.

Em relação ao processo científico do nosso trabalho, as cinco dimensões de pesquisa se desenvolvem em processos interligados e dialéticos iniciando com a dimensão epistemológica ou pelo levantamento do estado do conhecimento para se construir o objeto de estudos. Nessa dimensão o objeto é delineado e problematizado. Vale destacar que a busca por referências teóricas iniciais no decorrer do trabalho, após a dimensão técnica ou desenho metodológico e a dimensão morfológica onde se apresentam os dados/resultados, a escolha do corpo teórico é revisada para suspensão, troca ou acréscimo de outras fontes literárias. Essas podem ser reorganizadas mesmo pós a dimensão analítico-conclusiva onde os dados tratados são cruzados com a literatura. As dimensões exigem um ir e vir na literatura e nos dados até que se suspenda, mesmo que temporariamente, as dúvidas ou se elaborem novas hipótese para futuros trabalhos.

Em nossa dimensão técnica adotou-se a técnica do desenho pela sua potencialidade lúdica e informal de tratar um tema que são favoráveis à pesquisa das representações sociais. Trata-se, portanto de uma técnica facilitadora de materialidade ao pensamento/conhecimento sobre um determinado objeto/tema e de se compreendê-lo, sob a luz da Psicologia (BLEGER, 1972, 1975; KAES, 1976; AIELLO-VAISBERGER, 1995).

O desenho, segundo Atkison (2008), define como uma prática semiótica, tanto quanto para Barthes (2002), assinalando valor enquanto modo de transcrição gráfica de uma imagem mental que nos permite compreender a imagem consciente e inconsciente (latente) do grupo.

Segundo Penn (2002) Barthes (1972) trouxe uma nova leitura e compreensão dirigida à análise de imagens num processo inverso ao estabelecido por Saussure (1915) na linguística, ou seja, que se utilize tal análise, como uma descrição pragmática.

RPI Revista de Pesquisa Interdisciplinar, Cajazeiras, v. 1, Ed. Especial, 02-19, set/dez. de 2016. 
Neste sentido, vemos as imagens, objetos e comportamentos significando, mas não de forma independente e sim, vinculado à linguística, onde a imagem ancora-se no texto que a acompanha.

Neste caminho é que se evidencia a diferença entre linguagem e imagem, onde a imagem é, geralmente, passiva de inúmeras interpretações e assim, o texto a acompanha no sentido de dar-lhe sua única mensagem.

O estudo foi destinado aos profissionais da PSF/ESF/SF que tratam de tabagistas no Estado do Rio de Janeiro os quais foram informados sobre o Termo de Consentimento Livre e Esclarecido tendo o mesmo sido assinado por cada um dos profissionais. Procurou-se saber deles o que conhecem sobre o tabagista e como o tratam. Daí, levantar a coleta de dados ter sido realizada por meio de técnica projetiva com uso de desenhos e pequenos textos elaborados sob a perspectiva de Visca (1994) para compreender o fenômeno da configuração das representações sociais. Cabe esclarecer que os sujeitos foram abordados individualmente, em diferentes encontros (3) de capacitação para profissionais de saúde das ESF.

A escolha destas pessoas foi dada em razão da abertura da pesquisadora com os profissionais da PSF/ESF/SF em estudo, uma vez que faz parte da equipe onde foi realizada a pesquisa. A amostra foi de 30 profissionais sendo $97 \%$ do sexo feminino e na idade entre 25 a 50 anos, todos com nível superior e de especialização. Somente um dos profissionais com Mestrado. Assim, os entrevistados foram escolhidos contingencialmente, no município de Resende, RJ. $92 \%$ são do sexo feminino e $8 \%$ do sexo masculino. Entre todos circulam algumas funções e são elas: 4 médicos, 16 enfermeiros, 6 agentes comunitários de saúde, 3 dentistas e 1 fisioterapeuta.

Na aplicação da Técnica Projetiva foi adotada uma dinâmica para realização de um desenho de modo descontraído onde o sujeito convidado a participar foi esclarecido da pesquisa, do objetivo, dos riscos e benefícios, com a assinatura do Termo de Consentimento Livro e Esclarecido (TCLE). O procedimento proposto nesta pesquisa assegurou ao sujeito da pesquisa a devida proteção da sua identidade, impedindo o estigma e a utilização das informações em prejuízo de terceiros e da comunidade. Todos os participantes realizaram a técnica projetiva e todo o material foi utilizado para os fins propostos no protocolo de pesquisa, preservando, ainda, a autoestima e o prestígio dos sujeitos da pesquisa.

No desenho, as consignas foram: 1) desenhar um tabagista; 2) dar título ao desenho; 3) escrever 5 palavras que caracterizem um tabagista; 4) circular a mais significativa.

RPI Revista de Pesquisa Interdisciplinar, Cajazeiras, v. 1, Ed. Especial, 02-19, set/dez. de 2016. 
Uma vez com os dados em mãos, os analisamos sob à luz da psicologia social de Moscovici (1979), na sua perspectiva psicossocial (Vala, 2004), verificando a frequência com que estes sujeitos demonstraram os conhecimento sobre os tabagistas.

Nesta psicologia observa-se, além da forma, a imagem constituída de percepção, além da estética, seja de produção ou recepção. São dados para compreender o processo perceptivo. A percepção é entendida aqui como uma elaboração ativa, uma complexa experiência que transforma a informação recebida em atitudes. Neste contexto, a valoração e a forma de enfrentamento da doença pode ser trabalhada/analisada.

Os dados serão apresentados em forma de imagens e textos na dimensão morfológica.

\section{Resultados}

A análise da Técnica Projetiva do Desenho permitiu criarmos as categorias de análises ilustrada na Tabela de Análise da Técnica Projetiva que estão apresentados no decorrer deste texto e distribuídas nas tabelas 1 e 2 .

Na primeira etapa da referida Técnica foi solicitado que se desenhasse um tabagista que nos possibilitou gerar duas grandes categorias denominadas de "Cenário" e de "Figuras".

A grande categoria "Cenário" foi subdividida em outras duas categorias medianas denominadas em "Desenho/cenário" e "Desenho/dimensão". Na mediana que descreve o desenho dentro de um cenário geramos outras 4 categorias menores com os seguintes critérios:

- Simples em que o cenário observado foi em relação a descrição de até dois elementos na composição do desenho.

- Complexo 1 - enquadra os desenhos complexos com 2 a 3 elementos na composição do desenho.

- Complexo 2 - agregou os desenhos com mais de 3 elementos na composição do desenho.

- Lugar - aponta o lugar onde o desenho é pensado (casa, hospital, outro)

RPI Revista de Pesquisa Interdisciplinar, Cajazeiras, v. 1, Ed. Especial, 02-19, set/dez. de 2016. 
Na categoria mediana denominada "Desenho/dimensão" encontramos três categorias menores. Estas descrevem o desenho em "pequeno", "médio" e "grande". A categoria de tamanho foi medida segundo as dimensões da área do desenho demarcada por um retângulo de 10x12. Assim, o desenho sendo menor que a metade da área estabelecida seria considerado "pequeno". Se o desenho ultrapassasse a linha mediana, sem contudo, ter ultrapassado mais de um terço da área do desenho seria categorizado "médio". E os desenhos que chegassem a medida superior a um oitavo da área seriam considerados "grandes".

A grande categoria "Figuras" foi subdividida em duas categorias médias denominadas de "Corpo humano" e de "Objeto".

$\mathrm{Na}$ categoria média "Corpo humano" encontramos 11 pequenas categorias que se explicam por elas mesmas. A saber: 1. Homem; 2. Mulher; 3. Adolescente; 4. Objetado; 5. Animal; 6. Saudável; 7. Doente; 8. Sentimento; 9. Alegre; 10. Triste e; 11. Sem expressão

$\mathrm{Na}$ categoria média "Objeto" encontramos duas categorias menores "Cigarro" e "Outro".

$\mathrm{Na}$ segunda consignia "escrever cinco palavras que caracterizem o tabagista e circular a mais significativa" criamos as categorias de acordo com as palavras e/ou expressões usadas gerando categorias únicas que caracterizavam os desenhos em "Características Físicas", "Características Psíquicas” e "Atitudes”.

As categorias da Técnica Projetiva, como apontado anteriormente são descritas neste tópico por partes.

A primeira descrição é em relação aos sujeitos, segue a descrição do desenho com as suas categorias "Cenário" e depois em relação a "Figuras".

A descrição na categoria "Desenho/cenário" é multifacetada, ou seja, o tabagista é desenhado em três cenários, onde não apresentam um lugar definido, exceto, os seguintes: cemitério (3 indicações); hospital (1indicação); campo (1 indicação) e; casa (1 indicação).

A categoria "Cenário" é ilustrada na tabela 1.

Tabela 1- Distribuição das categorias dos desenhos da categoria "Cenário" sobre o Tabagista dos Profissionais do PSF/ESF/SF de Resende, RJ.

1 - Desenhar um tabagista.

RPI Revista de Pesquisa Interdisciplinar, Cajazeiras, v. 1, Ed. Especial, 02-19, set/dez. de 2016. 


\begin{tabular}{|c|c|c|c|c|c|c|}
\hline \multicolumn{7}{|c|}{ CENÁRIO } \\
\hline \multicolumn{4}{|c|}{ Desenho/cenário } & \multicolumn{3}{|c|}{ Desenho/dimensão } \\
\hline $\begin{array}{l}\text { Simples } \\
\text { (até dois } \\
\text { elementos) }\end{array}$ & $\begin{array}{c}\text { Complexo } 1 \\
\text { (de } 2 \text { a } 3 \\
\text { elementos) }\end{array}$ & $\begin{array}{c}\text { Complexo } 2 \\
\text { (mais } 3 \\
\text { elementos) }\end{array}$ & LUGAR & Pequeno & Grande & Médio \\
\hline $13 \%$ & $21 \%$ & $67 \%$ & & $40 \%$ & $33 \%$ & $27 \%$ \\
\hline
\end{tabular}

A descrição em relação à Categoria "Desenho/dimensão" apresenta-se distribuído de modo equitativo, sendo 12 desenhos na dimensão "pequena"; 10 na "grande" e 8 na "média". Sendo que quanto maior o desenho, maior foi a sua complexidade (Figura 1). E quanto menor o desenho menor a sua complexidade (Figura 2).

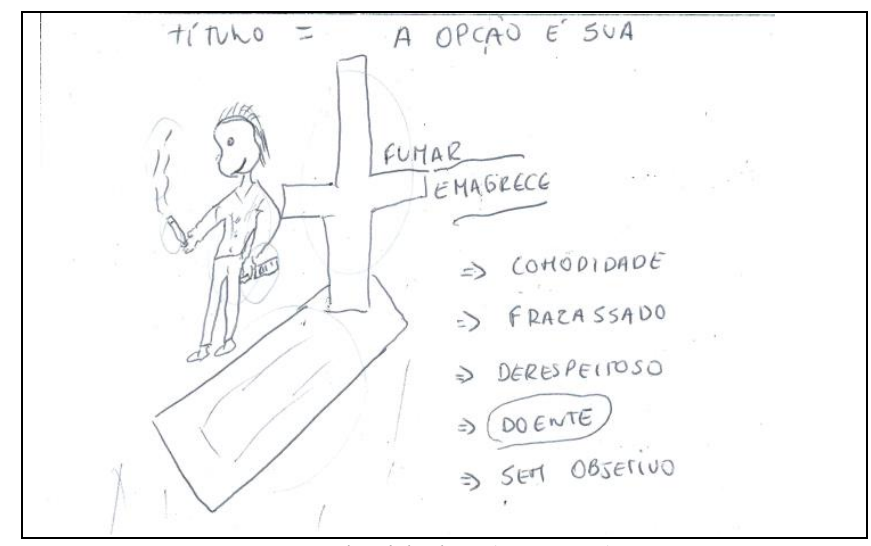

Fig. 1 - "Complexidade" (09_F_E)

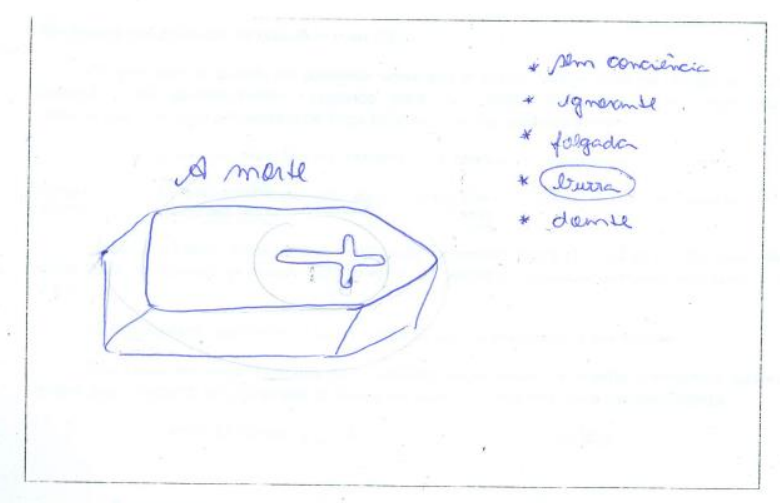

Fig. 2 - "Cemitério" (06_F_ACS)

Em relação à distribuição da categoria "Figura" temos as categorias menores "Corpo Humano" e a "Objeto", representados na Tabela 2.

RPI Revista de Pesquisa Interdisciplinar, Cajazeiras, v. 1, Ed. Especial, 02-19, set/dez. de 2016. 
Tabela 2- Distribuição das categorias dos desenhos da categoria "Figuras" sobre o Tabagista dos Profissionais do PSF/ESF/SF de Resende, RJ.

\begin{tabular}{|c|c|c|c|c|c|c|c|c|c|c|c|c|}
\hline \multicolumn{13}{|c|}{1 - Desenhar um tabagista. } \\
\hline & \multicolumn{12}{|c|}{ FIGURAS } \\
\hline & \multicolumn{10}{|c|}{ Corpo humano } & \multicolumn{2}{|c|}{ Objeto } \\
\hline 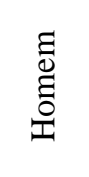 & $\frac{\overline{\mathrm{d}}}{\stackrel{\bar{\Xi}}{\Sigma}}$ & $\begin{array}{l}\frac{0}{0} \\
\frac{0}{0} \\
\frac{0}{0} \\
\frac{0}{0} \\
\frac{0}{4}\end{array}$ & $\begin{array}{l}\frac{8}{\pi} \\
\frac{\pi}{0} \\
\frac{0}{0}\end{array}$ & 丞 & 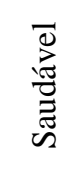 & 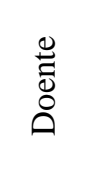 & 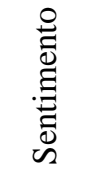 & $\begin{array}{l}\frac{0}{0} \\
\stackrel{0}{\mathbb{Z}} \\
\end{array}$ & 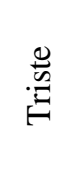 & 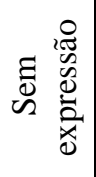 & 壹 & 苞 \\
\hline $27 \%$ & $23 \%$ & $50 \%$ & $20 \%$ & $3 \%$ & $70 \%$ & $30 \%$ & $80 \%$ & $53 \%$ & $20 \%$ & $27 \%$ & $80 \%$ & $20 \%$ \\
\hline
\end{tabular}

A categoria "Corpo Humano" em apreciação destaca a figura relacionada aos adolescentes (50\%), ilustrada na figura 3 .

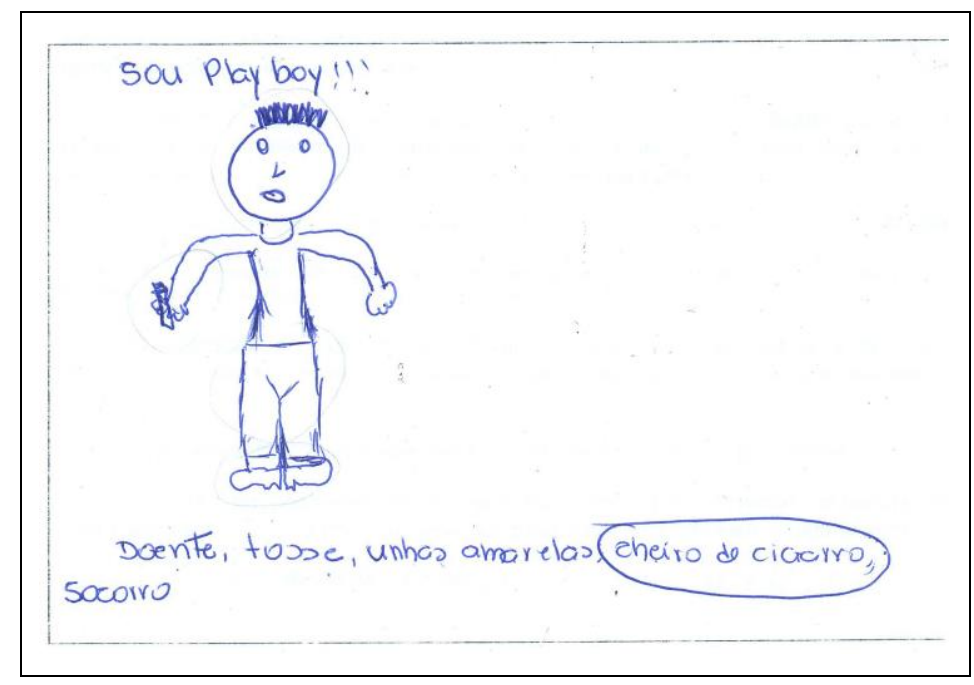

Fig3. "Jovem" (011_F_E)

A subcategoria "Doença" e "Saúde" tem como maioria (70\%) os aspectos de sujeitos "saudáveis".

Ainda tratando da categoria "Figura", os "Sentimentos" apresenta nos resultados que $80 \%$ desenharam o tabagista com algum tipo de sentimento. E destes 53\% são alegres (figura 5); $20 \%$ expressam tristeza e $27 \%$ sem expressão.

Vale apontar que os tamanhos dos desenhos não estão correlacionados aos sentimentos. Contudo a complexidade do desenho, como ilustra a figura 6 está associada ao sentimento de "alegria".

RPI Revista de Pesquisa Interdisciplinar, Cajazeiras, v. 1, Ed. Especial, 02-19, set/dez. de 2016. 
A sub-categoria "objeto" teve o cigarro como objeto que aparece em $80 \%$ dos desenhos, e em $20 \%$ são desenhos de animais e/ou caixão.

Em relação ao "Título do Desenho" denota-se três categorias. A saber: "Visão negativa"; "visão positiva" e "visão neutra".

Assim, 84\%, dos entrevistados possuem uma perspectiva negativa sobre o tabagista em que o mesmo é descrito, por exemplo, como "Burro" (02_F_M); "Pé na cova" (025_F_ACS); “O vicio controlando a vida” (012_F_E).

Somente um total de $11 \%$ entrevistados possuem uma perspectiva positiva sobre o tabagista. Exemplos: "Prazer" (03_F_M); "Estou de boa" (015_F_E); "Estou de bem com a vida" (22_F_E)

Um mínimo de 5\% dos profissionais possuem uma visão neutra do tabagista. Exemplo: "O resultado" (027_F_ACS).

Quanto as formas de expressão encontramos 4 categorias. São elas “adjetivos"(18\%); “metáforas" (27\%); "fala pelo outro" (17\%) e; “expressão chavão”(38\%).

Entre os adjetivos encontra-se "Folgado" (08_F_D); "A tabagista ansiosa" como "metáfora" (10_F_E) e ; "Tabagista crônico" (19_F_E) como exemplo de "palavra chavão".

Em relação à segunda consigna "Escrever cinco (5) palavras que caracterizem um tabagista" as categorizações do desenho apresentaram 4054 evocações e destas geramos três categorias denominadas de características "Físicas", "Psíquicas" e de "Atitudes".

A análise das mesmas foi de dois modos. A primeira análise foi em razão da frequência de uso da palavra ou expressão e a segunda centrou-se na ordem de aparecimento das palavras, trazendo a tona uma representação oposta entre as mesmas, como mostramos a seguir.

$\mathrm{Na}$ categoria "Características Físicas" temos 141 evocações (33\%), sendo 41 expressões geradas pela similitude, ou seja, sinônimos e aproximações de verossimilhança. Ex.:velho, idoso, envelhecido.

Na categoria denominada de "Características Psíquicas" encontramos 143 evocações (33\%), gerando 53 expressões de verossimilhança. Ex. ansioso, ansiedade, agitado.

Em relação à categoria "Atitude" temos 148 evocações (34\%), com 53 expressões por semelhança ou aproximação de sentido. Ex.: câncer, morto e doente. 
Destas características $97 \%$ são negativas e somente $3 \%$ ressaltam características positivas como "esperançoso"; "prazer" e "socorro".

Em relação à terceira consigna "Circular a mais significativa para você", também geramos as mesmas categorias anteriores, ou seja, características "Físicas" (43\%), como as mais significativas; "Psíquicas" (33\%) e; de "Atitudes" (23\%). Estas são apresentadas na Tabela 6.

Cabe destacar que aqui todas as características físicas são negativas. Observamos que estas características "significativas", apontadas pelos Profissionais representam o contrário das evocações anteriores, onde foram analisadas todas as palavras, sem destaque para a "mais significativa".

Tabela 3 - Distribuição das categorias, segundo as evocações de palavras mais significativas da Técnica Projetiva que caracterizam o Tabagista pelos Profissionais do PSF/ESF/SF -Resende, RJ.

\begin{tabular}{c|c|c}
\hline $\begin{array}{c}\text { CARACTERISTICAS } \\
\text { FISICAS }\end{array}$ & $\begin{array}{c}\text { CARACTERISTICAS } \\
\text { PSIQUICAS }\end{array}$ & ATITUDES \\
\hline 13 & 10 & 7 \\
\hline $\mathbf{4 3 \%}$ & $\mathbf{3 3 \%}$ & $\mathbf{2 3 \%}$ \\
\hline
\end{tabular}

A distribuição das evocações de palavras "mais significativas" dos profissionais expressaram $43 \%$ com grande inclinação para as relacionadas às características físicas tais como doente, morte e feio.

Apesar desta correlação "negativa" do tabagista há evocações de palavras que expressam a esperança.

Quando se cruza as categorias do desenho é possível observar a representação imagética de um sujeito que está num lugar distante do Profissional do PSF/ESF/SF de Resende, no estado do Rio de Janeiro. Em nenhum desenho aparece o sujeito em diálogo com este profissional. Dados que são analisados no próximo capítulo.

\section{Discussão}

RPI Revista de Pesquisa Interdisciplinar, Cajazeiras, v. 1, Ed. Especial, 02-19, set/dez. de 2016. 
A história do tabaco parece guardar força na memória coletiva dos profissionais do $\mathrm{PSF} / \mathrm{ESF} / \mathrm{SF}$ de Resende, quando observamos que estes associam o tabagismo entre o glamour e a decadência do ser tabagista.

Tal fato mostra-se evidente a partir da percepção de que há duas décadas, numerosos hábitos e comportamentos eram tidos como corriqueiros e sequer despertavam interesse entre os profissionais de saúde, entre eles, o tabagismo, que era considerado "um vício elegante" (FOCCHI, 2008).

Em contrapartida e de acordo com Laranjeiras (2004), o que hoje se apresenta é que fumar ha muito deixou de ser um ato de glamour e é, atualmente, o comportamento que mais produziu mortes na história da humanidade.

Seguindo nosso arcabouço teórico aportamos nas Representações Sociais e nelas também balizamos a construção deste trabalho no sentido de que temos acompanhados por Arruda (2002) a afirmativa comum que a representação social é a forma de saber prático que liga o sujeito a um objeto.

Desta forma nos permitimos pensar na relação que usuário e profissional estabelecem em busca da adesão e cessação do vicio, mas que permeada pelos sentidos variados, esta relação se fragiliza diante das representações sociais com características de similitude que o grupo de profissionais constitui acerca do tabagista.

Jodelet (2003) já nos ensinou, nessa perspectiva, o quanto é preciso se destacar que a representação social é sempre representação de alguém e de alguma coisa, tendo com seu objeto, relação de simbolização e de interpretação. Deparamo-nos, então, diante da constatação de que o tabagista foi, finalmente, inserido dentro do processo saúde e doença.

A concepção deste citado processo, como pontuado anteriormente, tem evoluído de maior vinculação com a doença e morte para maior vinculação com a qualidade de vida e assim, passando de um sentido negativo para um sentido positivo (Mendes, 1995). Podemos ter inserido, portanto, neste contexto, o tabagista que além de ser alvo de morte, tem comprometida, enquanto sobrevive, sua qualidade de vida e a das pessoas que o rodeiam e isso, quer dizer, tanto de forma ativa quanto passiva.

As representações sociais instituídas ao longo da história como nos esclarece Almeida (2011), nos traz a possibilidade de consideração da vida cotidiana e, nela, seus múltiplos enredamentos. Neste cotidiano ousamos verificar as representações sociais destes 
profissionais da saúde sobre os tabagistas e a valoração do mesmo, enquanto indivíduo ativo na busca de sua aprendizagem e cessação do uso do tabaco e de seus derivados.

As representações sociais investigadas nesta pesquisa indicaram uma concepção de tabagista com maior vinculação com a doença e a morte em detrimento da vinculação com a qualidade de vida. Cabe destacar que as palavras "mais significativas" destacaram as características físicas negativas. Cremos seja por conta da cultura de focar no diagnóstico "doença" e não no doente, ser com possibilidades.

A proposta de enfrentamento da doença deveria ser de vinculação inversa e assim, passando de um sentido negativo para um sentido positivo como já pontuou Mendes (1995) em seu estudo da relação entre o processo de produção social associado a acesso a bens e serviços econômicos e sociais e a qualidade de vida.

Portanto, neste contexto, o tabagista deve ser entendido na sua totalidade, ou seja, pensado como sujeito vivente de uma sociedade consumista, numa cultura específica, com um núcleo familiar e uma subjetividade a ser compreendida e não um doente "burro"; "pé na cova"; "sem amor próprio". Deve ser entendido, então, como uma pessoa "ansiosa"; "dependente" e "esperançosa". Assim, a ação do profissional será nesta direção de atenção, cuidado e respeito. São representações negativas que podem levar ao abandono ao tratamento.

É com uma perspectiva humanizada da saúde que acreditamos favorecer a percepção dos profissionais frente aos tabagistas e possamos fortalecer a cultura em prol da qualidade de vida. Afinal, são os profissionais que devem compreendê-lo dentro do contexto sócio histórico e que além de ser alvo de morte, tem comprometida, enquanto sobrevive, sua qualidade de vida e a das pessoas que o rodeiam e isso, quer dizer, tanto de forma ativa quanto passiva. Daí sinalizar ao tabagista que há saúde a ser alimentada e esta depende de uma parceria humanizada entre o profissional e o tabagista.

\section{Considerações Finais}

As representações sociais instituídas ao longo da história nos traz a possibilidade de consideração da vida cotidiana e, nela, seus múltiplos enredamentos. Neste cotidiano, ousamos verificar as representações sociais dos profissionais do PSF/ESF/SF de Resende sobre o tabagista, com a finalidade de valoração do trabalho destes profissionais frente aos tabagistas, na mesma relação valorativa, uma vez que este, também deve ser tratado como

RPI Revista de Pesquisa Interdisciplinar, Cajazeiras, v. 1, Ed. Especial, 02-19, set/dez. de 2016. 
indivíduo ativo na busca de sua aprendizagem e cessação do uso do tabaco e de seus derivados.

O projeto desenvolvido nos permitiu compreender as representações sociais dos tabagistas para estes profissionais, como sendo pessoas jovens na figura de homem e adultos doentes na figura de mulheres.

As representações sociais levantadas nos permitiu delinear três categorias e dois modos. As características denominadas de "características físicas"; "características psíquicas" e "atitudes". O primeiro modo ou análise foi sobre o total de palavras evocadas nos desenhos e sobressaiu a categoria das "atitudes" com 34\% do total. Em relação a mais significativa a "característica física" destacou-se com $43 \%$ das opções. Assim, as representações sociais dos profissionais estudados marcam a ideia de um tabagista com atitudes "negativas" e as “características físicas” depreciativas.

Os resultados guardam correlação com a teoria, sobre a faixa etária que mais chama a atenção tanto para os órgãos públicos de saúde como para os profissionais estudados, "O Adolescente".

Assim, apontamos que a figura do "Adolescente" que sobressaiu nos desenhos vem ao encontro das ações do Ministério da Saúde em relação ao foco escolhido pelas indústrias do tabaco que acentuam suas estratégias de divulgação e de modificação do cigarro com uso de aromas e químicos que possam atrair esta faixa etária.

A localização dos cigarros perto de doces e balas dos cigarros em lojas de conveniência também reforça o exposto acima.

As mulheres diferentemente de décadas atrás se tornam consumidoras e dependentes do cigarro e revelam um grau de dificuldade na adesão e cessação do vício.

Percebemos, também, que os profissionais que desenvolvem suas atividades no Programa de Combate ao Tabagismo ainda carregam ideias e valores que merecem uma revisão em prol do fortalecimento deste grupo para efetivar a adesão e a cessação deste vicio pelo tabaco.

Deste modo, nossa suspeição de que os tabagistas aderem pouco ao Programa, pode estar relacionado à forma como estes são representados pelos profissionais pode estar dificultando esta adesão, acentua-se e nos instiga a novos estudos, ampliando o grupo, agora junto aos próprios tabagistas. Aqui marcamos nossa proposta de continuidade de pesquisas.

RPI Revista de Pesquisa Interdisciplinar, Cajazeiras, v. 1, Ed. Especial, 02-19, set/dez. de 2016. 
Acrescenta-se que na ampliação do estudo será possível comprovar a eficiência do nosso produto que é uma capacitação específica por meio de um dispositivo auto-instrucional em forma de CD-ROM que possibilite aos profissionais do PSF/ESF/SF uma visão mais humanizador do tabagista.

Enfim, os profissionais estudados denotaram uma dificuldade em perceber o tabagista como ser em tratamento que necessita de um discurso mais humanizador, ai a validade de nosso produto como recurso instrucional em prol de novas percepções sobre o tabagista como homem em busca do direito a vida saudável.

\section{REFERÊNCIAS}

AIELlO-VAISBERGER, T. M. J. O uso de procedimentos projetivos na pesquisa de representações sociais: projeção e transicionalidade. Psicol. USP [online]., vol.6, n.2, pp. 103-127. , 1995.

ALMEIDA, A. M. de O.; S, Santos, M. de F. de S; Trindade, Zeidi, Araújo. Teoria das Representações Sociais: 50 anos. Brasília: TechnoPolitik, 2011.

ARRUDA, A. (2002).Teoria das Representações Sociais e Teorias de Gênero - Cadernos de Pesquisa, n. 117, novembro. . (2008).

ATKINSON,D. (2008).Pedagogy Against the State Internacional Journal of Art and Design Education, Vol 27.

BARTHES, R. (1975).Para/ou onde vai a literatura. In: VÁRIOS. Escrever... para quê? Para quem? Lisboa, Edições 70.

BAUER, M. W.; GASKELL, G. Pesquisa qualitativa com texto, imagem e som: um manual prático. Petrópolis: Editora Vozes, 2003.

BLEGER, J.Temas de psicologia. Buenos Aires, Nueva Vision, 1972.

Brasil. (2001).Ministério da Saúde. Instituto Nacional de Câncer - INCA. Coordenação de Prevenção e Vigilância (CONPREV). Abordagem e Tratamento do Fumante - Consenso 2001. Rio de Janeiro: INCA.

Ministério da Saúde. Instituto Nacional de Câncer (Brasil). (2011). Organização

Pan-Americana da Saúde. Pesquisa especial de tabagismo - PETab: relatório Brasil / Instituto Nacional de Câncer. Organização Pan-Americana da Saúde. - Rio de Janeiro: INCA.

Ministério da Saúde. Instituto Nacional do Câncer INCA., 2001 Coordenação de Prevenção e Vigilância (CONPREV). Abordagem e Tratamento do Fumante Consenso (2001). Rio de Janeiro: INCA, 38 p.

RPI Revista de Pesquisa Interdisciplinar, Cajazeiras, v. 1, Ed. Especial, 02-19, set/dez. de 2016. 
Ministério da Saúde. Portaria do Ministério da Saúde $\mathbf{n}^{\circ} 1.035$ de 31 de maio

de 2004. Amplia o acesso à abordagem e tratamento do tabagismo para a rede de atenção básica e de média complexidade do Sistema Único de Saúde.

Ministério da Saúde. Secretaria Nacional de Assistência à Saúde. Instituto

Nacional de Câncer. (1997) Coordenação Nacional de Controle do Tabagismo e Prevenção Primária de Câncer (Contapp). Ajudando seu paciente a deixar de fumar. Rio de Janeiro: INCA, , $52 \mathrm{p}$.

FOCCHI G.R.A. Tabagismo: dos fundamentos ao tratamento - Rev. psiquiatr. clín. vol.35 no.1. São Paulo, 2008.

IGLESIAS, V., Cavada, G., SILVA, C. ; Caceres, D. (2007). Early tobacco and alcohol consumption as modifying risk factors on marijuana use. Revista de saúde publica, 41, 51722, 2007.

JODELET, D. (Org.). As Representações Sociais. Rio de Janeiro: EdUERJ, 2003.

Experiência e representações sociais. In MENIN, Maria Suzana De S.; Shimizu, A. de M. Experiência e Representação Social: questões teórico e metodológicas. São Paulo: Casa do Psicólogo, 2005.

KAES, R..El aparato psíquico grupal: Construciones de grupo. Espanha: Garnica Editor, 1976.

LARANJEIRAS, R. - In: RAW, Martin. (2009). Parar de fumar é possível -Publifolha - 2 ed. São Paulo, 2004.

MALBERGIER, A., .Tabagismo: dos fundamentos ao tratamento. Rev. psiquiatr. clín. vol.35 no.1, São Paulo. 2008.

MENDES, E. V. (Org.). Distrito sanitário: o processo social de mudança das práticas sanitárias do Sistema Único de saúde. 4. ed., Rio de Janeiro: Abrasco, 1995.

MOSCOVICI, S. (2003). Representações sociais: investigações em psicologia social. Rio de Janeiro, Vozes.

(1979). Comunicação apresentada ao Colóquio sobre as Representações

Sociais. Paris, EHESS, 8-10 jan.

NOVIKOFF, C. (2002). As Representações Sociais Acerca dos Adolescentes: perspectivas e práticas pedagógicas em construção. Dissertação (Mestrado em Educação), Universidade do Estado do Rio de Janeiro.

.(2010).Desafios da Práxis Educacional à Promoção Humana na Contemporaneidade. Rio de janeiro, Reproarte.

. (2006). NOVIKOFF, C. Os Caminhos da Construção Pedagógica: Instituindo o

Ser Professor In: Escola Competente. 1 ed.Rio de Janeiro : Wak Editora, v.01, p. 207-230.

Organização Mundial de Saúde (OMS). Classificação Estatística Internacional de Doenças

e Problemas Relacionados à Saúde - Décima Revisão - (CID 10).

RPI Revista de Pesquisa Interdisciplinar, Cajazeiras, v. 1, Ed. Especial, 02-19, set/dez. de 2016. 
PENN, G. Análise semiótica de imagens paradas. In: BAUER, Martin W.; GASKELL, G. Pesquisa qualitativa com texto, imagem e som: um manual prático.Petrópolis: Editora Vozes, p.319-342, 2003.

SANTAELLA, L. O que é semiótica? São Paulo: Brasiliense, 1983.

SAUSSURE, F. de. Curso de Lingüística Geral. 11. ed. São Paulo: Cultrix, s/d. 1915

VALA, J.; Monteiro;.M. B. Psicologia Social. Lisboa: Fundação Calouste Gulbenkian, 2004.

VISCA, J. L. Clinica psicopedagógica: epistemología convergente. 2. ed. Buenos Aires: Edição do autor, 1994. 